\title{
Oresme, Lucain et la « voix de sorcière »
}

\section{Béatrice Delaurenti}

\section{(2) OpenEdition}

Journals

Édition électronique

URL : https://journals.openedition.org/crm/764

DOI : $10.4000 / \mathrm{crm} .764$

ISSN : 1955-2424

\section{Éditeur}

Honoré Champion

\section{Édition imprimée}

Date de publication : 30 décembre 2006

Pagination : 169-179

ISSN : 1272-9752

Référence électronique

Béatrice Delaurenti, « Oresme, Lucain et la « voix de sorcière » », Cahiers de recherches médiévales [En ligne], 13 | 2006, mis en ligne le 27 novembre 2009, consulté le 15 décembre 2022. URL : http:// journals.openedition.org/crm/764 ; DOI : https://doi.org/10.4000/crm.764 


\section{ris}

\section{Oresme, Lucain et la « voix de sorcière »}

Dans la littérature enfantine, le personnage de la sorcière se caractérise par un balai, un chat noir, un chapeau pointu, une verrue ... et une voix particulière, caverneuse ou glaçante. Cet attribut vocal appartient à l'imaginaire contemporain : la voix émane du corps de la sorcière, elle le prolonge, elle augmente son pouvoir. Quel serait le ressort de la puissance de cette voix ? Quelles en sont les sources? Sur ces questions, les réflexions d'un théologien du XIV ${ }^{\mathrm{e}}$ siècle apportent un éclairage intéressant. Le topos de la «voix de sorcière » a été développé par Nicole Oresme avant que la sorcellerie ne devienne une préoccupation majeure du monde occidental. La puissance terrifiante de la voix de sorcière n'est pas une invention du $\mathrm{XX}^{\mathrm{e}}$ siècle.

Nicole Oresme (1320-1382) est une personnalité centrale de la vie politique et intellectuelle du $\mathrm{XIV}^{\mathrm{e}}$ siècle européen. Il entra au collège de Navarre comme boursier en 1348, sans doute après un premier cursus à la faculté des arts de l'Université de Paris ; il obtint le grade de Docteur en théologie, devint Maître du Collège de Navarre en 1356 et poursuivit une carrière d'enseignant. En 1361, il renonça à son poste au Collège de Navarre pour suivre la carrière ecclésiastique, et fut nommé évêque de Lisieux en $1377^{1}$. L'œuvre de Nicole Oresme est rédigée tantôt en latin, principalement pour les textes antérieurs à 1361, tantôt en français dans les années 1360-1370. Elle couvre des domaines variés : la science, la philosophie, l'économie, la lutte contre l'astrologie et la pastorale ${ }^{2}$.

La thématique de la «voix de sorcière» est abordée dans un traité de géométrie et de philosophie naturelle, le De configuratione qualitatum et motuum; Oresme l'a composé alors qu'il était au Collège de Navarre, entre 1351 et $1362^{3}$. Il y déve-

\footnotetext{
${ }^{1}$ Peu de recherches ont été faites sur la vie de Nicole Oresme depuis la biographie initiale de F. Meunier, Essai sur la vie et les ouvrages de Nicole Oresme, Paris, 1857. Il reste plusieurs zones d'ombre dans sa biographie. Sur les différentes dates de sa carrière, voir F. Neveux, "Nicole Oresme et le clergé normand du XIV siècle ", Autour de Nicole Oresme. Actes du colloque de l'Université de Paris XII, éd. J. Quillet, Paris, Vrin, 1990, p. 9-36.

${ }^{2}$ Pour une présentation des œuvres de Nicole Oresme, voir J. Gautier-Dalché, « Oresme et son temps", Nicolas Oresme, tradition et innovation chez un intellectuel du XIVe siècle, éd. P. Souffrin et A. Segonds, Paris, Belles Lettres, 1988, p. 8-9. La liste complète de ses écrits se trouve dans A.-D. Menut, «A provisional bibliography of Oresme's writings ", Mediaeval Studies, 28, 1966, p. 279-99 et 31, 1969, p. 346-347. Plus récemment, B. Patar a complété cette liste par les références des manuscrits et des éditions ainsi qu'une esquisse de chronologie des œuvres d'Oresme : Nicolas Oresme, Expositio et Quaestiones in Aristotelis De Anima. Édition, étude critique, Louvain/Paris, Peeters, 1995, p. 15*-29*.

${ }^{3}$ Aucun des quatorze manuscrits du traité ne donne de date. D'après l'éditeur du traité, Oresme aurait rédigé le De configuratione pendant son enseignement à la faculté des arts, c'est-à-dire avant qu'il obtienne le grade de Maître en théologie (1356), ou peut-être avant qu'il quitte le collège de Navarre (1362). Cf. M. Clagett, Nicolas Oresme and the medieval geometry of qualities and motions. A treatise on the uniformity and difformity of intensities known as Tractatus de configurationibus qualitatum et motuum, Madison, University of Wisconsin Press, 1968, p. 122-125.
}

Cahiers de Recherches Médiévales, 13, 2006 
loppe une nouvelle doctrine scientifique pour expliquer l'ensemble des phénomènes relevant du corps et de l'âme. Les qualités et les mouvements de chaque corps, dit-il, peuvent être représentés par des figures géométriques; les configurations géométriques obtenues sont propres à chaque corps, elles donnent à voir l'ensemble des qualités naturelles d'un corps et ses divers effets. La doctrine des configurations explique de cette manière les phénomènes corporels, même lorsqu'ils sont prodigieux et mystérieux.

Dans le second livre du traité, Oresme applique la doctrine des configurations aux phénomènes qui relèvent de la magie. Le pouvoir des sons et, par extension, celui des mots, sont considérés comme une des racines de l'efficacité magique. C'est à propos de l'idée de pouvoir des mots, ou virtus verborum, que l'auteur évoque la puissance de la voix humaine. L'exemple s'inscrit dans une réflexion sur les incantations employées par les magiciens. Oresme veut montrer que la puissance de ces formules ne provient pas de la signification des mots, mais d'une virtus d'un autre ordre ${ }^{4}$. La voix du magicien possède une puissance qui n'est pas attachée au sens des mots :

Que ces choses n'agissent pas par la force de leur signification mais par le pouvoir de leur structure et figuration est évident [...] parce que [les magiciens (magi)] n'utilisent pas toujours un son significatif mais murmurent des sons déformés par une difformité étrange et inhabituelle, difforme et dissemblable à la voix humaine ordinaire. Lucain, en parlant d'une certaine incantation, a dit ainsi : «Et alors sa voix, plus puissante que n'importe quelle drogue pour enchanter les dieux de Léthé, murmura d'abord des chuchotements, en dissonant et dans une grande discorde avec la voix humaine. L'aboiement du chien et le hurlement du loup étaient dans cette voix. $»^{5}$

Certaines incantations sont incompréhensibles: la signification des mots n'est donc pas un principe d'action. Oresme choisit le poète Lucain (39-65 ap. JC) pour illustrer cet argument; il cite un épisode de son épopée sur la guerre civile, $D e$ bello civili. Lucain y fait le portrait d'une prophétesse (vates), Erictho, qui prédit l'avenir en invoquant les défunts :

Et alors sa voix, plus puissante que toutes les herbes pour évoquer les dieux du Léthé, murmure d'abord des sons discordants et bien différents du langage humain. Elle a l'aboiement du chien et le hurlement des loups, la plainte du hibou tremblant, de la strige nocturne, le grincement ou le grognement des bêtes sauvages, le siffle-

\footnotetext{
${ }^{4}$ Sur la position d'Oresme sur les incantations et la virtus verborum, voir Béatrice Delaurenti, Virtus verborum. Débats doctrinaux sur le pouvoir des incantations au XIII et au XIV siècle, Paris, EHESS, thèse de doctorat, 2004, à paraître aux éditions du Cerf au printemps 2007.

${ }^{5}$ Nicole Oresme, De configuratione, II, 33, éd. M. Clagett, op. cit. p. 368 : «Quod autem ista fiant non ex vi significationis sed virtute formationis et figurationis sonorum patet [...] quia [magi] non semper utuntur voce significativa sed murmurant nescio quid quadam extranea et insolita difformitate sonora difformi - ymmo difformi atque dissimili humane voci communi. Unde Lucanus de quadam incantatione loquens sic dicebat: "Tunc vox Letheos cunctis potentior herbis excusare (i. e. excantare) deos confundit murmura primum dissona et humane multum discordia lingue. Latratus habet illa canum gemitusque luporum »(les interventions dans le texte sont celles de l'éditeur).
} 
ment du serpent, elle rend les battements de l'eau qui se brise sur les écueils, le bruissement des forêt et le tonnerre de la nuée qui crève : tant de choses ont formé une seule voix. ${ }^{6}$

La voix de la prophétesse ressemble à des cris d'animaux, à des bruits naturels. Ce sont des sonorités effrayantes et peu harmonieuses : aboiement, hurlement, grincement, sifflement ou bruissement, le vocabulaire est étendu. Il évoque les vibrations sonores du monde nocturne. Cette séquence auditive n'est pas le produit d'une fiction littéraire, elle correspond plutôt à la description d'un rituel magique. L'imitation de sons animaux et végétaux est inscrite, en effet, dans certaines traditions magiques gréco-latines. Lucain pourrait s'en être inspiré7. Mais pour Oresme, l'essentiel n'est pas là. C'est la puissance sonore de cette voix qui importe. Le théologien prolonge le texte de Lucain en commentant deux versets d'Isaïe sur la divination :

Et dans Isaie il est dit : «Cherche les pythonisses et les devins qui hurlent dans leurs incantations », etc., car hurler est différent du langage ordinaire. [...] Et alors dans Isaïe il est dit : "Cherche les pythonisses» etc., certains livres ayant «Cherche les ventriloques» et une autre lecture a «Cherche les pythonisses qui parlent depuis la terre et pleurent depuis le ventre [de la terre] »; car ailleurs dans le même livre il est dit en reproche «Ta voix pourra venir de la terre comme celle de la pythonisse, et hors du sol ton discours sera murmuré $»^{8}$

Oresme propose de remplacer le terme de devineresse (python) par celui de ventriloque (ventriloquus). L'apport de ces citations bibliques tient précisément au vocabulaire: l'incantation apparaît comme un hurlement (stridor) qui s'écarte du langage courant, un murmure (mussitatio). Dotée d'une certaine autonomie, elle est composée de sons inarticulés venus des entrailles du devin. C'est cela, ajoute Oresme, qui caractérise la voix du magicien :

\footnotetext{
${ }^{6}$ Lucain, De bello civili, VI, v. 685-88, texte établi et traduit par A. Bourgery, Paris, Belles Lettres, 1976, $5^{\text {e }}$ éd., p. 35: Tunc uox Lethaeos cunctis pollentior herbis / excantare deos confundit murmura primum / dissona et humanae multum discordia linguae. / Latratus habet illa canum gemitusque luporum / quod trepidus bubo, quod strix nocturna queruntur / quod strident ululantque ferae, quod sibilat anguis ; / exprimit et planctus inlisae cautibus undae / siluarumque sonum fractaeque tonitrua nubis. / Tot rerum uox una fuit.

${ }^{7}$ Voir A. Bourgery, «Lucain et la magie », Revue des études latines, 6, 1928, p. 299-313, et L. Baldini-Moscadi, «Osservationi sull'episodo magico del VI libro della Farsaglia di Lucano », Studi italiani di filologia classica, 48, 1976, p. 140-199 (en part. p. 173-174).

${ }^{8}$ Nicole Oresme, op. cit. p. 368-370: Et in Ysaia dicitur : "Querite a phitonibus [i. e. pythonibus] et a divinis qui strident in [in] cantationibus suis » etc; stridere enim non est communis locutio. [...] Unde in Ysaia ubi dicitur: "Querite a phitonibus» etc., quidam libri habent "Querite a ventriloquis» et alia littera habent "Querite a phitonibus qui de terra loquuntur et de ventre clamitant"; nam et alibi in eodem libro in obprobrium dicitur, "erit quasi phitonis de terra vox tua, et de humo eloquium tuum mus[s]itabit» (les interventions sur le texte latin sont celles de l'éditeur). Is 8, 19: Et cum dixerint ad vos quaerite a pythonibus et a divinis qui stridunt in incantationibus suis, numquid non populus a Deo suo requirit pro vivis a mortuis ? et Is 29, 4 : Humiliaberis de terra loqueris et de humo audietur eloquium tuum, et erit quasi pythonis de terra vox tua et de humo eloquium tuum mussitabi.
} 
Parfois il arrive que [le magicien (magus)] forme des sons variés intérieurement et qu'une voix inappropriée et semblable à une trompette résonne, parfois involontairement, et alors on suppose qu'un démon parle en lui ou sous la terre. ${ }^{9}$

Les citations d'Isaïe et les commentaires d'Oresme montrent comment le théologien conçoit la voix lorsqu'elle est porteuse d'une puissance. C'est une voix inhumaine et bestiale, une voix qui vient de l'intérieur, des entrailles de la terre ou de celles d'un être humain. Elle véhicule des paroles dont on ne peut saisir le sens. Tantôt murmure, tantôt chuchotement, tantôt sifflement ou hurlement sauvage, c'est une voix discordante et dissonante que le magicien entend sortir de sa poitrine sans vraiment la maîtriser. Cette voix est communément rapportée aux démons, précise Oresme : c'est cela qu'il entend réfuter en montrant que la voix possède en ellemême une puissance naturelle.

\section{Un projet naturaliste}

C'est précisément la qualité animale et végétale de la voix qui intéresse Nicole Oresme dans le De configuratione. La voix d'Erictho est plus proche d'un son brut que d'une parole intelligible qui serait adressée à un interlocuteur. La voix humaine est en principe l'instrument de la raison, elle marque la différence entre les hommes et animaux, tandis que la voix ensorcelante paraît rejoindre le monde physique ou animal. Elle serait un maillon intermédiaire entre les bruits et la parole. Cet entre-deux tient une place de choix dans la démonstration d'Oresme. Il s'inscrit dans une démonstration sur les causes des procédés magiques.

Le thème de la puissance de la voix est central dans ce chapitre du De configuratione. Le pouvoir des sons et des mots (virtus sonorum seu verborum), dit l'auteur, est un des ressorts de la magie. Et parmi toutes les sortes de sons, c'est la voix humaine, «sonus vox et maxime vox humana», qui est la plus apte à posséder une grande virtus ${ }^{10}$. Dès le début du chapitre, une citation de Jérôme met en avant la force particulière de la voix humaine. Dans une lettre envoyée au prêtre Paulin de Nole en 394-96, Jérôme dispense certains conseils sur la formation chrétienne du prêtre. Il montre l'importance des voyages et donne l'exemple de saint Paul qui allait de ville en ville pour prêcher l'Évangile. Jérôme estime qu'une prédication de vive voix est supérieure à un prêche écrit pour obtenir la conviction et la conversion de l'interlocuteur. Il ajoute cette remarque :

Il y a je ne sais quelle énergie cachée dans la voix vivante ; transmise aux oreilles des disciples, elle y résonne plus fortement. ${ }^{11}$

\footnotetext{
${ }^{9}$ Nicole Oresme, op. cit. p. 368 : Aliquando contingit quod varios sonos format [magus] ad intra et resonat inconveniens vox quasi tubalis, quam emittit ab ymo pectore aliquotiens non sponte, et tunc putatur quod demon loquatur in illo vel sub terra.

${ }^{10}$ Id., p. 366 : Tertium fundamentum artis magice consistit in sonorum seu verborum virtute. Ad hanc autem efficatiam potissime aptus est sonus vox et maxime vox humana.

${ }^{11}$ Saint Jérôme, Lettres, lettre 53, §2, Paris, Belles Lettres, 1953, texte établi et traduit par

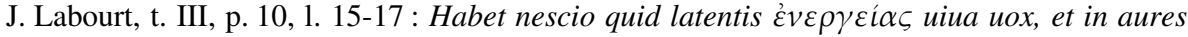
discipuli de auctoris ores transfusa fortius insonat.
} 
Le timbre et l'éclat de la voix du prédicateur possèdent une énergie, une force, un pouvoir plus percutants que la lecture d'un message rédigé ; cette énergie latente (latens) ne demande qu'à être mise en acte. Jérôme utilise le terme grec

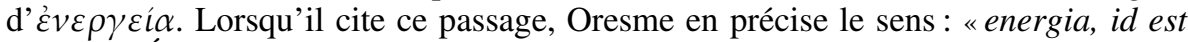
virtus $»^{12}$. Énergie est un des sens du latin virtus, qui désigne à la fois la vigueur, la force d'âme et la qualité morale. Le rapprochement lexical entre $\varepsilon \dot{v} \varepsilon \rho \gamma \varepsilon \dot{c} \alpha$ et virtus permet à Oresme de relier l'energia vocis du prédicateur de Jérôme à sa propre réflexion sur la virtus verborum.

L'analyse de la voix conduit donc aux mots : la voix, décrite dans la diversité de ses manifestations, est considérée comme le support des mots. Un vers des Amours d'Ovide vient illustrer l'importance des mots en magie. Le poète met en scène une sorte de magicienne entremetteuse, experte en relations amoureuses. Elle a des pouvoirs occultes, elle connaît les arts magiques, les vertus des herbes et des astres, et «ses longues incantations savent ouvrir la masse de la terre $»^{13}$. Oresme évoque ensuite, une nouvelle fois, les incantations d'Erictho, si puissantes qu'il ne lui est pas nécessaire de recourir à un autre poison : "l'esprit périt sous les incantations, sans qu'aucun poison absorbé ne l'ait infecté de son venin $»^{14}$. La prophétesse additionne parfois le pouvoir du poison à celui de l'incantation :

Souillant Philippe de ses incantations et l'aspergeant de ses philtres sinistres, [elle] lui défendit de faire passer ailleurs les combats. ${ }^{15}$

Ces deux citations, Ovide et Lucain, ouvrent une fenêtre sur l'usage des incantations en magie. Oresme présente l'incantation comme l'outil par excellence de ceux qui pratiquent l'art magique : un outil de qualité, particulièrement efficace, un acte universel que l'on utilise en dernier recours et qui agit autant sur les hommes que sur les animaux. Le but d'Oresme n'est pas de fournir une justification à des pratiques condamnées et condamnables. Mais il voit dans l'usage particulier que les magiciens font des incantations le signe d'une préséance des formules par rapport aux autres méthodes de l'art magique. Dès lors, son objectif est de montrer que les mots agissent par leurs sonorités et non par leur signification.

Quelle serait la cause naturelle de la virtus verborum? L'interprétation d'Oresme se fonde sur la doctrine de la configuration des qualités. Dans la pensée

\footnotetext{
${ }^{12}$ Nicole Oresme, op. cit. p. 366 : De qua ait Ieronymus : "Habet enim nescio quid latentis energie vive vocis actus" (energie, id est virtutis).

${ }^{13}$ Ovide, Les amours, I, §9, 1. 15-18, Paris: Belles Lettres, texte établi et traduit par H. Bornecque, 1989 : Oculis quoque pupila duplex / Fulminat et gemino lumen ab orbe micat. / Euocat antiquis proauos atauosque sepulcris / Et solidam longo carmine findit humum. Cf. Nicole Oresme, op. cit. p. 366 : De quadam enim vetula dicit Ovidius : "Et solidam longo carmine findit humum ».

${ }^{14}$ Lucain, De bello civili VI, v. 457, op. cit. p. 24 : Mens hausti nulla sanie polluta ueneni, / excantata perit. Cf. Nicole Oresme, op. cit. p. 366: Quandoque autem magi hoc solo sine iuvamine alterius radicis immutant hominum mentes. Inde ait Lucanus : "Mens hausti nulla sanie polluta veneni, excantata perit », etc.

${ }^{15}$ Lucain, De bello civili VI, v. 580, p. 30 : Pollutos cantu dirisque venefica succis / conspersos uetuit transmittere bella Philippos. Cf. Nicole Oresme, op. cit. p. 366 : Quandoque etiam cum hoc iuvat se magus alia radice. Ideo dicit Lucanus : "Pollutos cantu diris[que] venefica succis", etc.
} 
médiévale, un objet ou un phénomène physique se caractérise par un ensemble de qualités. Oresme propose de représenter graphiquement, pour un objet donné, la répartition de ces qualités au moyen d'une figure géométrique, une configuratio. Chaque corps est déterminé par une configuration qui reflète sa structure qualitative; de cette configuration découlent les propriétés physiques du corps considéré. La doctrine s'applique à tout objet sensible, en particulier aux sons : ils disposent de capacités particulières en vertu de la configuration de leurs qualités ${ }^{16}$. Dans ce schéma, l'homme conserve une marge de manœuvre: il lui est possible d'agir sur un objet en modifiant la configuration de ses qualités, de telle sorte qu'il peut ensuite en tirer des effets merveilleux. C'est ainsi que les sons produisent parfois des phénomènes extraordinaires, des mirabilia ${ }^{17}$.

La démonstration s'applique aussi aux mots en tant qu'ils représentent une forme sonore particulière : les effets prodigieux de certains mots ont une cause naturelle. L'interprétation d'Oresme est naturaliste; elle assimile les formules d'incantations à des productions sonores, ce qui met hors jeu toute considération démoniaque. La thématique de la puissance de la voix sert de support à une réflexion plus vaste sur les pouvoirs de la nature : elle permet à Oresme de mettre en évidence les forces naturelles qui interviennent vraiment lorsqu'une personne croit agir par magie.

\section{La prophétesse, le magicien et la vetula}

Les analyses d'Oresme font de la voix le vecteur d'une puissance redoutable, une puissance naturelle. Il reste à identifier les détenteurs d'un tel pouvoir : qui parle derrière cette voix ? Trois personnages se profilent dans ce tableau.

Il y a d'abord la devineresse de Lucain, la sombre Erictho (tristis Erictho), présentée comme une prophétesse thessalienne (Thessala vates) dans le De bello civili $^{18}$. Erictho cherche au royaume des morts un interprète des enfers pour obtenir des réponses à ses questions. Elle pratique la divination en invoquant l'esprit des morts, c'est une nécromancienne au sens premier du terme. Dans les sources médiévales, cependant, la nécromancie (necromantia) est souvent confondue avec la nigromancie (nigromantia). Ce terme désigne une divination fondée sur l'invocation des démons au moyen de pratiques magiques. La nécromancie antique, centrée sur les morts, aurait laissé la place à une nigromancie centrée sur les démons. L'évolution du rituel et du vocabulaire est le résultat d'un processus complexe d'influences réciproques. Cependant, dans l'usage, nécromancie et nigromancie sont employés au Moyen Âge comme des synonymes, ils désignent une magie centrée sur le contrôle des esprits ${ }^{19}$. Lorsque Nicole Oresme cite Erictho, il ne fait pas le

\footnotetext{
${ }^{16}$ Sur la doctrine des configurations, voir A. Maier, « La doctrine d'Oresme sur les configurationes intensionum ", dans Ausgehendes Mittelalter I: Gesammelte Aufsätze zur Geistesgeschichte des 14. Jahrhunderts, Rome, 1964, p. 335-352, et M. Clagett, op. cit., p. 14-50.

${ }^{17}$ Nicole Oresme, op. cit. p. 366 : Non est difficile videre - ymmo probabile est - quod naturaliter vel artificiose possit alicuius soni difformitas taliter figurari quod habebit potentiam aliquid ad extra mutandi, et precipue in animali illo quod afficitur per auditum ab huiusmodi sono.

${ }^{18}$ Lucain, De bello civili VI, v. 605, 628, 640, 651, op. cit.

${ }^{19}$ Cf. J.-P. Boudet, «La genèse médiévale de la chasse aux sorcières. Jalons en vue d'une relecture. », Le mal et le diable. Leurs figures à la fin du Moyen Âge, dir. N. Nabert, Paris
} 
portrait d'une nécromancienne pratiquant la divination: il décrit une nigromancienne pratiquant la magie.

Le magicien (magus) est le second personnage qui apparaît dans le chapitre de Nicole Oresme sur le pouvoir de la voix. L'ars magica fait l'objet d'une longue digression dans le traité De configuratione. Oresme analyse les fondements de la magie, il dénonce l'art de l'illusion que pratiquent les magiciens, leurs tromperies et la crédulité du public ${ }^{20}$. La magie et l'astrologie ont connu un succès grandissant dans les milieux lettrés dans la deuxième moitié du $\mathrm{XIV}^{\mathrm{e}}$ siècle, c'est dans ce contexte que Nicole Oresme a rédigé ce texte. Mais son combat contre les magiciens et les astrologues est surtout postérieur au traité De configuratione. En 1361, après avoir quitté le Collège de Navarre, le théologien s'est lié d'amitié avec le dauphin, le futur Charles V. Plus tard, à la demande de Charles V, il a effectué des missions officielles et traduit en français plusieurs traités d'Aristote ${ }^{21}$. Or le roi entretenait des astrologues à la cour et manifestait lui-même un grand intérêt pour la science des astres. Les écrits polémiques d'Oresme attaquent les prétentions des magiciens et des astrologues. Les pages du De configuratione consacrées à l'art magique ont été un prélude à ces écrits contre les sciences divinatoires et la magie ${ }^{22}$.

Beauchesne, 1996, p. 38, et Astrologie, divination et magie dans l'Occident médiéval (XII $X V^{e}$ siècle). Essai de synthèse, Paris, Université de Paris I-Panthéon-Sorbonne, 2003, à paraître aux Publications de la Sorbonne en 2006, p. 390.

${ }^{20}$ Pour une analyse du regard que porte Oresme sur la magie, les démons et la nature, voir S. Caroti, «Eléments pour une reconstruction de la philosophie de la nature dans les Quodlibeta de Nicole Oresme", Autour de Nicole Oresme. Actes du colloque de l'Université de Paris XII, éd. J. Quillet, Paris, Vrin, 1990, p. 85-118 ;M. Clagett, op. cit. ; B. Hansen, Nicole Oresme and the marvels of nature. A study of his De causis mirabilium with a critical edition, translation and commentary, Toronto, Pontifical Institute of Mediaeval Studies, 1985; E. Paschetto, «Linguaggio e magia nel De configurationibus di Nicole Oresme » et «Demoni e astri dans les Quodlibeta », Demoni e prodigi. Note su alcuni scritti di Witelo e di Oresme, Turin, 1978, p. 7-80 ; B. Patar, op. cit. ; J. Quillet, «L'imagination selon Nicole Oresme», Archives de philosophie, 50, 1987, p. 219-227, et «Enchantements et désenchantement de la Nature selon Nicole Oresme», Mensch und Natur im Mittelalter, Berlin/New-York, De Gruyter, 1991, vol. I, p. 321-329 ; L. Thorndike, A History of Magic and Experimental Science during the first thirteen Centuries of our Area, New York, Mac Millan Company, 1934, vol. III, chap. 25-27 ; ainsi que les actes des colloques Nicolas Oresme, tradition et innovation chez un intellectuel du XIV ${ }^{e}$ siècle, op. cit., et Autour de Nicole Oresme. Actes du colloque de l'Université de Paris XII, éd. J. Quillet, Paris, Vrin, 1990.

${ }^{21}$ Sur les relations entre Oresme et Charles V (1338-1380, roi en 1364), voir E. Grant, « Nicole Oresme, Aristotle's on the Heavens, and the court of Charles V » Texts and Contexts in Ancient and Medieval Science, Leiden : Brill, 1997, p. 187-207 et J. Cadden, "Charles V, Nicole Oresme and Christine de Pizan : unities and uses of knowledge in fourteenth-century France», id., p. 208-244, ainsi que J. Quillet, Charles V, le Roi lettré : Essai sur la pensée politique d'un règne, Paris, Librairie Académique Perrin, 1984, p. 105-114.

${ }^{22}$ Sur les écrits anti-astrologiques d'Oresme, voir S. Caroti, «La critica contro l'astrologia di Nicole Oresme e la sua influenza nel medioevo e nel Rinascimento », Atti dell'Accademia Nazionale dei Lincei, s. VIII, vol. XXIII, 6 (1979), p. 545-685 ; B. Hansen, op. cit. p. 4 note 6 et p. 17-25; Max Lejbowicz, «Chronologie des écrits anti-astrologiques de Nicole Oresme. Étude sur un cas de scepticisme dans la deuxième moitié du XIV ${ }^{\mathrm{e}}$ siècle », dans Autour de Nicole Oresme, op. cit., p. 119-176. 
Il faudrait ajouter un troisième personnage à ce panorama : la vetula. Dans les textes médiévaux, la vetula est un personnage complexe et ambivalent, parfois bénéfique, souvent maléfique. Elle incarne généralement l'archétype de la vieille femme dotée de pouvoirs occultes et néfastes; elle est citée pour illustrer la futilité des pratiques magiques et la bêtise de ceux qui s'y adonnent ${ }^{23}$. Elle est en quelque sorte l'ancêtre de la sorcière de la fin du Moyen Âge, souvent représentée comme une femme âgée, veuve et marginale. Nicole Oresme évoque ce personnage peu recommandable dans ses Quodlibeta (1370): il rapporte une discussion qu'il aurait eue avec une vieille femme accusée de pratiquer la magie. La vetula est décrite comme un personnage faible, craintif, manipulé par les juges et dépassé par les accusations qu'on lui impute ${ }^{24}$.

La vetula est également présente, en filigrane, dans le De configuratione. Certes, Oresme ne parle pas explicitement de vetula lorsqu'il décrit Erictho et sa voix maléfique. Le combat qu'il mène contre ceux qui pratiquent la magie ne vise pas directement ce personnage, moins influent que les magiciens et les imposteurs qui pratiquent la fausse persuasion et abusent de la confiance qui leur est accordée. Cependant la silhouette de la vetula se profile derrière les exemples qu'Oresme emprunte à Ovide et à Lucain et qui sont tous des exemples féminins. En outre, la magicienne à la voix puissante s'apparente à une vetula par son ambivalence et par la manière dont elle exploite, sans le savoir, le pouvoir naturel des sons.

Le De configuratione est, à notre connaissance, le seul texte de l'époque scolastique qui fasse une telle part au pouvoir de la voix. Nicole Oresme donne au pouvoir de la voix une origine antique et païenne, il sert de relais aux idées de Lucain sur les qualités animales et végétales de la voix. La voix puissante que décrit Nicole Oresme n'est pas exactement une «voix de sorcière »; ce serait plutôt la voix d'une nécromancienne- nigromancienne ou celle d'un magicien, éventuellement celle d'une vetula. Cette analyse du pouvoir de la voix fournit néanmoins une base philosophique au topos de la «voix de sorcière».

\section{Les inquisiteurs et le pouvoir de la voix}

L'analyse d'Oresme a-t-elle eu une postérité ? Le De configuratione est rédigé dans les années 1351-62, plusieurs décennies avant que la lutte contre les sorcières ne devienne une obsession européenne. Le point de départ de la chasse aux sorcières se situerait dans les années 1258-60, au moment où les fondements idéologiques de la répression se mettent en place. De 1300 à 1330, le nombre des procès pour magie et sorcellerie est faible; les deux tiers de ces procès ont un caractère politique. Cette période marque néanmoins une étape importante dans l'histoire de la sorcellerie, puisqu'elle correspond au moment où prennent forme les thèmes démonologiques qui caractérisent la fin du Moyen Âge. Les démons, jadis intégrés au monde naturel, gagnent une sorte d'autonomie dans la pensée

\footnotetext{
${ }^{23}$ Sur la vetula, voir J. Agrimi, C. Crisciani, «Savoir médical et anthropologie religieuse. Les représentations et les fonctions de la vetula $\left(\mathrm{XIII}^{\mathrm{e}}-\mathrm{XV}^{\mathrm{e}}\right.$ s.) », Annales Économies, Sociétés, Civilisations, 48/5 (1993), p. 1281-1308.

${ }^{24}$ Nicole Oresme, Quodlibeta, questio 44, Napoli, Bibl. Nazionale Vittorio Emmanuele III, XI C 84, fol. 120v. Sur ce texte, voir Béatrice Delaurenti, op. cit., chap. 11 et l'édition critique de la questio 44 en Annexe 4.
} 
monde naturel, gagnent une sorte d'autonomie dans la pensée occidentale ${ }^{25}$. Mais la nouvelle obsession démoniaque n'a pas eu d'incidence immédiate sur les procès en sorcellerie, qui se sont raréfiés de 1330 jusqu'à l'époque d'Oresme. C'est seulement au tournant du $\mathrm{XIV}^{\mathrm{e}}$ et du $\mathrm{XV}^{\mathrm{e}}$ siècle que la chasse aux sorcières a vraiment commencé : entre 1375 et 1435 , le nombre de procès de sorcellerie augmente de façon spectaculaire $^{26}$. Le tournant décisif se situe en 1435-1440 : ces années-là sont marquées par l'élaboration d'un discours construit et abouti sur les sorcières et sur le sabbat $^{27}$.

Le stéréotype de la sorcière émerge au début du $\mathrm{XV}^{\mathrm{e}}$ siècle en même temps que celui du sabbat. La sorcière des inquisiteurs médiévaux se caractérise principalement par le pacte qu'elle passe avec le diable, par sa participation au sabbat et par sa pratique du vol nocturne. La «voix de sorcière» ne fait pas partie de cette panoplie, elle n'est pas stigmatisée par les inquisiteurs ${ }^{28}$.

Toutefois, la voix est mentionnée par Henri Institoris et Jacques Sprenger lorsqu'ils font le portrait de la sorcière dans le Malleus Maleficarum. Ce célèbre manuel était destiné à guider l'inquisiteur dans sa démarche. Publié à Strasbourg en 1486, il fut régulièrement réédité, et demeura une référence dans les procès de sorcellerie $^{29}$. Une des questions du manuel demande «pourquoi plus de femmes que

${ }^{25}$ Cf. A. Boureau, Satan hérétique. Naissance de la démonologie dans l'Europe médiévale (1260-1350), Paris, Odile Jacob, 2004.

${ }^{26}$ Cette chronologie de la chasse aux sorcières est fondée sur J.-P. Boudet, «La genèse médiévale de la chasse aux sorcières. Jalons en vue d'une relecture. », op. cit., p. 40-52, et Astrologie, divination et magie dans l'Occident médiéval (XII ${ }^{e}-X V^{e}$ siècle), op. cit. p. 330-360. Une chronologie de l'ensemble de la chasse est proposée par B. P. Levack dans La grande chasse aux sorcières en Europe aux débuts des temps modernes, Paris, Champ Vallon, 1991, $1^{\mathrm{e}}$ éd. 1987, p. 181-185. En 1435 commencerait une première période de chasses massives, accompagnée par la publication des traités de sorcellerie ; après une certaine stabilisation au début du XVI ${ }^{\mathrm{e}}$ siècle, l'apogée de la chasse est située entre 1580 et 1675 ; à partir de cette date, elle connaît un déclin progressif jusqu'en 1750.

${ }^{27} \mathrm{Cf}$. M. Ostorero, "Folâtrer avec les démons": Sabbat et chasse aux sorciers à Vevey (1448), Lausanne, Université de Lausanne, Faculté des Lettres, Section d'histoire, Cahiers Lausannois d'histoire médiévale 15, 1995, p. 188. Sur la sorcellerie au Moyen Âge, la bibliographie est vaste. Voir en particulier A. Boureau, « Le sabbat et la question scolastique de la personne », Le sabbat des sorciers en Europe (XVe-XVIII siècles). Actes du colloque international ENS Fontenay-Saint-Cloud, 4-7 novembre 1992, dir. N. Jacques-Chaquin et M. Préaud, Grenoble, Jérôme Millon, 1993, p. 33-46 ; N. Cohn, Démonolâtrie et sorcellerie au Moyen Âge, fantasmes et réalités, Paris, Payot, 1982, $1^{\text {ere }}$ éd. 1975; C. Ginzburg, Le sabbat des sorcières, Paris, Gallimard, 1992, $1^{\text {ere }}$ éd. 1989; S. Houdard, Les sciences du diable : quatre discours sur la sorcellerie, Paris, Cerf, 1992.

${ }^{28}$ Voir par exemple L'imaginaire du sabbat. Edition critique des textes les plus anciens $(1430$ ca. - 1440 ca.), textes réunis par M. Ostorero, A. Paravicini-Bagliani, K. Utz Tremp, Lausanne, 1999. Les cinq textes édités dans ce recueil ne font aucune allusion à la voix des sorcières. Sur le stéréotype de la sorcière, voir N. Cohn, op. cit., p. 129-133.

${ }^{29}$ L'édition princeps du Malleus est celle de Strasbourg, 1486 ; 15 éditions ont suivi jusqu'en 1520. 
d'hommes [sont] engagées dans la superstition $»^{30}$. La réponse est l'occasion de souligner les mauvais effets de la voix féminine :

Écoutons encore ceci au sujet d'une autre des particularités [de la femme], la voix. Menteuse par nature, elle l'est dans son langage : elle pique tout en charmant. D'où la voix des femmes est comparée au chant des Sirènes, qui par leur douce mélodie attirent ceux qui passent et les tuent. Elles tuent en effet car elles vident la bourse, elles enlèvent les forces, elles contraignent à perdre Dieu ${ }^{31}$.

L'explication prend appui sur la légende des sirènes, exposée par Homère dans l'Odyssée et par Ovide dans les Métamorphoses ${ }^{32}$. Pour Institoris et Sprenger, la sorcière est avant tout une femme et la voix est un de ses atouts : elle n'est pas vraiment terrifiante, mais c'est une voix féminine, chargée des plaisants pouvoirs de la femme. La sonorité de cette voix fonde le pouvoir de la sorcière. Elle charme l'auditeur, elle l'ensorcelle; le diable ici n'intervient pas. Le concept de «voix de sorcière » est réduit à un attribut du sexe féminin.

Cette formulation du Malleus maleficarum est faible et décevante, sans rapport avec le renom du topos. La «voix de sorcière » n'est rien d'autre qu'une voix féminine, charmante et enjôleuse. Elle n'a pas d'autre origine que l'épisode des sirènes, relayé par la misogynie des inquisiteurs médiévaux. Les analyses d'Oresme, la référence à Lucain sont restées lettre morte au $\mathrm{XV}^{\mathrm{e}}$ siècle. La notion de «voix de sorcière » n'avait pas de consistance à l'époque des procès en sorcellerie.

Il reste donc Lucain, Oresme et cette tentative de justifier la force des incantations par le pouvoir de la voix. Notre sorcière enfantine, avec ses vibrations vocales, a d'abord été prophétesse et magicienne, nécromancienne et nigromancienne. Les deux personnages se mêlent et se répondent. L'une réveille les morts, l'autre interpelle les démons, mais leur voix puissante et redoutable est l'unique agent de leur pouvoir. Le topos de la «voix de sorcière » serait né de cette idée que la voix, la voix féminine en particulier, disposerait d'une puissance naturelle.

Comment expliquer le silence relatif des inquisiteurs? Pourquoi la «voix de sorcière » ne fait-elle pas partie du stéréotype ? La réponse tient peut-être aux particularités du regard que porte Oresme sur la magie. Car le théologien est étranger à la rhétorique des inquisiteurs. Sa position sur les mirabilia laisse peu de place aux démons. En montrant que les pratiques magiques sont inefficaces et que les magiciens sont des imposteurs, l'auteur du De configuratione montre en même temps que les démons, s'ils existent, sont ici inutiles. L'agent démoniaque est complètement

\footnotetext{
${ }^{30}$ Henri Institoris et Jacques Sprenger, Malleus Maleficarum, I, VI, Göttingen, fac. sim. de l'éd de Lyon, Claude Landry, 1620, p. 64 : ... cur in maiores multitudines reperiantur magis foemina superstitiosa quam viri, trad. A. Danet, Grenoble, Jérôme Millon, 1990, p. 175.

${ }_{31}^{31}$ Id. : Audiamus et aliam proprietatem per vocem. Nam sicut est mendax in natura, sic et in loquela, nam pungit, et tamen delectat. Unde et earum vox cantui Syrenarum assimilatur, quae dulci melodia transeuntes attrahunt et tamen occidunt. Occidunt quidem, quia ex marsupio evacuant, vires auserunt, et Deum perdere cogunt. Trad. A. Danet, op. cit., p. 180.

${ }^{32}$ Homère, Odyssée, Chant XII, v. 39-55 et 165-200, texte établi et traduit par Victor Bérard, Paris, Belles Lettres, 1992, p. 112-113 et 118-119; Ovide, Métamorphoses, V, v. 551-571, texte établi et traduit par G. Lafaye, Paris, Belles Lettres, 1985, p. 143.
} 
éliminé du débat. C'est pourquoi les remarques d'Oresme sur la voix ne se rapportent pas au personnage de la sorcière soumise aux démons: elles servent un autre projet, un projet naturaliste.

Oresme décrit une sorcière avant la lettre, une femme d'action qui manie les incantations avec énergie et bestialité. La voix est symptomatique du personnage, elle est un des fondements de son pouvoir. Ce pouvoir est détourné de toute interprétation démoniaque : la voix incarne un pouvoir issu de la nature, et non du diable. C'est la consistance sonore de la voix, bien plus que la signification qu'elle véhicule, que retient Nicole Oresme : par le biais de la voix et des sonorités qu'elle fait entendre, c'est le corps sorcier qui est mis en lumière. Il vient s'inscrire dans un champ qui n'est pas démoniaque, mais naturel.

Béatrice Delaurenti EHESS - Groupe d'Anthropologie Scolastique 